\title{
Pain-Relief Effects of Aroma Touch Therapy with Citrus junos Oil Evaluated by Quantitative EEG Occipital Alpha-2 Rhythm Powers
}

\author{
Tomomi Bohgaki ${ }^{1,2}$, Yoshitada Katagiri ${ }^{3}$, Makoto Usami ${ }^{2}$ \\ ${ }^{1}$ College of Nursing, Aichi Medical University, Aichi, Japan \\ ${ }^{2}$ Kobe University Graduate School of Health Sciences, Hyogo, Japan \\ ${ }^{3}$ Advanced ICT Research Institute, National Institute of Information and Communications Technology, \\ Tokyo, Japan \\ Email: bohgaki@aichi-med-u.ac.jp
}

Received December 1, 2013; revised December 29, 2013; accepted January 8, 2014

Copyright (C) 2014 Tomomi Bohgaki et al. This is an open access article distributed under the Creative Commons Attribution License, which permits unrestricted use, distribution, and reproduction in any medium, provided the original work is properly cited. In accordance of the Creative Commons Attribution License all Copyrights ( 2014 are reserved for SCIRP and the owner of the intellectual property Tomomi Bohgaki et al. All Copyright (C) 2014 are guarded by law and by SCIRP as a guardian.

\begin{abstract}
Aroma touch therapy is widely used in clinical fields for alleviating pain-related symptoms; however, few studies have reported the pain-relief mechanisms. The present study aimed to elucidate the analgesic effects of aroma touch therapy with Citrus junos oil based on the quantitative evaluation of deep brain network (DBN) activity using electroencephalogram (EEG) occipital alpha-2 rhythm (10 - $13 \mathrm{~Hz})$ powers. Experimental investigations were performed with 13 healthy volunteers using the cold pressor task for simulating chronic pain in three different sessions: a baseline session with no therapies, a control session with a touch therapy, and an aroma touch therapy. We have found for the first time that the interviewed pain ratings represented by Numeric Rating Scale (NRS) scores were strongly correlated with a DBN activity index, which was derived from the slow fluctuation components of occipital EEG alpha-2 rhythm powers. The correlation was characterized by a $\mathrm{V}$-shaped curve in the DBN activity index versus the pain rating, i.e., the NRS score, which provided the complete analgesic states $(\mathrm{NRS}=0)$ for some subjects under aroma touch therapy at an appropriate DBN activity index. Such analgesic states were not so strongly correlated with emotional valence. In conclusion, aroma touch therapy may directly modulate DBN activity so that pain-induced outcomes are minimized.
\end{abstract}

\section{KEYWORDS}

Aroma Touch Therapy; Chronic Pain; Encephalogram Occipital Alpha-2 Rhythm; Citrus junos (Yuzu) Oil

\section{Introduction}

Aromatherapy is a standard method used in complementary and alternative medicine [1-4]. Essential oils are utilized in aromatherapy, and they are synthesized from natural volatile organic compounds. Aromatherapy is characterized by low side effects $[5,6]$. This is of great advantage for clinical applications and is supported by a wide variety of practical implementations and evidence for the alleviation of symptoms related to higher-order brain dysfunctions, such as dementia [5,7-9], attention deficit hyperactivity disorders [10], and mood disorders [11-13], as well as for pain control $[14,15]$, stress man- agement, and health promotion [16-18]. Aromatherapy has also been applied to palliative care in hospice [19-23], and its validity has been demonstrated not only for maintaining a high quality of life (QOL) [24-27] but also for alleviating chronic pain associated with cancer [27].

Aromatherapy provides various practical therapies ranging from simple inhalation of an essential oil to a complicated aroma massage. Aroma touch is a type of massage, but the use of essential oils distinguishes it from original forms defined as "manual soft tissue manipulation including holding, causing movement, and/or applying pressure to the body", according to the American Massage Therapy Association [28]. Aroma touch 
therapy is equivalent to pediatric massage in which only mild pressure is applied. Hence, aroma touch therapy is considered safe and effective even for cancer patients in hospices [29] and has shown highly efficient effects of alleviating various symptoms associated with cancer [30-32]. Based on this assumption, we performed an advance clinical trial to examine the effects of aroma touch therapy on a cancer patient [33]. The results revealed that aroma touch therapy might improve QOL and reduce pain-related syndromes. Such clinical evidences are considered to support the recent increasing demands for aroma touch therapy in hospices.

Many studies have investigated chronic pain, as a target of aromatherapy, to elucidate neural mechanisms. To explain how chronic pain conditions are formed even in the absence of afflicted body parts, a concept of the Pain Matrix is proposed by Melzack [34]. This concept integrates pain-related cortical regions typically including the somatosensory area, anterior cingulate cortex, insular cortex, and medial prefrontal cortex to construct a pain network for pain perception. In the early stage of chronic pain studies, much interest was focused on thalamic activity, taking into account the anatomical structure that thalamus terminated the spinothalamic tract and transfered pain signals to the Pain Matrix [35]. Previous clinical studies utilizing neuroimaging techniques for cerebral regional blood flows [36] reported that thalamic activity is significantly decreased under chronic pain conditions. They also reported that completely pain-free states in patients with chronic pain can be obtained with thalamic stimulation [37-39]. From an electrophysiological view point, chronic pain conditions are related to dysfunctions of the thalamus, which are characterized as thalamocortical dysrhythmias, and those that can be detected in lowfrequency ranges of electroencephalogram (EEG) or mag-netoencephalogram signals [40-42]. On the basis of these clinical findings, it was assumed that the analgesic effects of aroma touch therapy can be attributed to thalamic activation. Hence, we were interested in detereming how thalamic activity was changed in pain-free states by aromatherapy.

The region related to the thalamus and limbic system is also strongly correlated with emotion. Hence, the emotional effects of aroma touch therapy on pain ratings must be considered to discuss the analgesic effects. According to the Pain Matrix model [43-45], pain sensation represented on the cortices can be emotionally modulated independently of thalamic activity. Pleasantness evoked by odor and touch reduces pain ratings and even provides pain-free states [46]. Such analgesic effects of aroma touch therapy are even considered as placebo effects given by higher-order brain functions [47-49]. Therefore, we were interested in determining whether thalamic activities or emotion changes were dominant.
The present study aimed to clarify the two issues of interest as mentioned above: the quantitative evaluation of thalamic activity under analgesic states and determination of a dominant factor from the two candidates of thalamic activity and emotional modulation evoked by aroma touch therapy. We therefore performed both neurophysiological and neuropsychological investigations on the analgesic effects of aroma touch therapy in healthy subjects who have no pain-induced reorganizational changes in the central nervous systems. In the neurophysiological investigation, we adopted information derived from occipital EEG alpha rhythms to quantitatively evaluate thalamic activity. EEG studies have a merit for investigating psychological aspects, such as pain or painrelated unpleasantness, compared with conventional studies with functional magnetic resonance imaging (fMRI) measurements, which could be stressful for subjects. A long history correlates with EEG alpha rhythms. Studies utilizing neuroimaging techniques revealed central neural correlates including the thalamus, which particularly attracted much interest because the results indicated that surface EEG reflected deep brain network (DBN) activities. Although whether the correlation is positive [50-52] or negative $[53,54]$ has been a matter of argument in published papers, a recent report $[55,56]$ concluded this issue through its findings that neural correlates with occipital EEG alpha rhythms depend on the frequencies of alpha rhythm power fluctuations. This report also gives the best suggestion that a region of the thalamus, associated with the brain stem and anterior cingulate cortex, is positively correlated with the slow fluctuation components of the alpha rhythm power. These neural parts related to the positive correlation are considered to form DBN. Hence, the evaluated slow components reflect DBN activity. We consider that this DBN activity corresponds to the thalamic activity evaluated by neuroimaging as mentioned above, because blood oxygenation level-dependent (BOLD) signals do not reflect any information related to the fast modulation components of the EEG alpha-2 rhythm powers.

\section{Materials and Methodology}

\subsection{Subjects}

Thirteen healthy Japanese volunteers including 10 females and 3 males (age, mean \pm SD: $21.2 \pm 1.36$ years) were enrolled in this study. We did not take the sex differences into account in this study. All of them provided written informed consent. We carefully checked their health conditions according to the medical history declared by themselves to eliminate participants having diseases such as a liver disease, kidney disease, neuropathy, heart disease, or even chronic pain. We also determined whether the participants were allergic to Yuzu essential oil using the conventional patch test. Table 1 
Table 1. Subject characteristics.

\begin{tabular}{|c|c|c|c|c|c|c|c|c|c|}
\hline \multirow{2}{*}{$\begin{array}{l}\text { Subject } \\
\quad \#\end{array}$} & \multirow[b]{2}{*}{ Sex } & \multirow{2}{*}{$\begin{array}{l}\text { Age } \\
\text { (year) }\end{array}$} & \multirow{2}{*}{$\begin{array}{l}\text { Height } \\
(\mathrm{cm})\end{array}$} & \multirow{2}{*}{$\begin{array}{l}\text { Weight } \\
\text { (kg) }\end{array}$} & \multirow{2}{*}{$\begin{array}{c}\mathrm{BMI} \\
\left(\mathrm{kg} / \mathrm{m}^{2}\right)\end{array}$} & \multicolumn{2}{|c|}{ STAI-form JYZ } & \multirow{2}{*}{$\begin{array}{c}\text { GSD } \\
\text { (depression } \\
\text { level) }\end{array}$} & \multirow{2}{*}{$\begin{array}{l}\text { Medical history and } \\
\text { Stress-associated symptoms }\end{array}$} \\
\hline & & & & & & $\begin{array}{l}\text { Trait Anxiety } \\
\text { (level) }\end{array}$ & $\begin{array}{l}\text { State Anxiety } \\
\text { (level) }\end{array}$ & & \\
\hline 1 & $F$ & 20 & 164 & 51 & 19 & 31 (I) & 52 (IV) & 37 (M) & \\
\hline 2 & $\mathrm{~F}$ & 25 & 155 & 66 & 27.5 & 60 (IV) & 36 (II) & $31(\mathrm{~L})$ & Persistence in thinking \\
\hline 3 & $\mathrm{~F}$ & 21 & 160 & 65 & 25.4 & 46 (III) & 43 (III) & 20 & $\begin{array}{l}\text { insistence on cleanliness } \\
\text { (chopsticks) }\end{array}$ \\
\hline 4 & $\mathrm{~F}$ & 21 & 158 & 50 & 20 & 41 (II) & 42 (II) & 29 & \\
\hline 5 & $\mathrm{~F}$ & 20 & 151 & 43 & 18.6 & 46 (III) & 30 (I) & 21 & overreact \\
\hline 6 & $\mathrm{~F}$ & 20 & 159 & 45 & 17.8 & 36 (II) & 46 (III) & 21 & $\begin{array}{l}\text { pulled muscle of a shoulder } \\
1 \text { week ago, pain continues }\end{array}$ \\
\hline 7 & $\mathrm{M}$ & 21 & 165 & 60 & 22 & 59 (IV) & 39 (II) & $33(\mathrm{~L})$ & \\
\hline 8 & $\mathrm{~F}$ & 20 & 160 & 58 & 22.7 & 29 (I) & 28 (I) & 24 & \\
\hline 9 & $\mathrm{~F}$ & 21 & 170 & 53 & 18.3 & 35 (II) & 38 (II) & 20 & \\
\hline 10 & $\mathrm{~F}$ & 21 & 160 & 52 & 20.3 & 33 (I) & $32(\mathrm{I})$ & 18 & \\
\hline 11 & $\mathrm{~F}$ & 22 & 167 & 64 & 23 & 47 (III) & 41 (III) & $31(\mathrm{~L})$ & particular about stimulation \\
\hline 12 & $\mathrm{M}$ & 22 & 174 & 64 & 21.1 & 34 (I) & 30 (I) & 18 & \\
\hline 13 & $\mathrm{M}$ & 22 & 173 & 75 & 25.1 & 30 (I) & 41 (II) & 17 & atopic dermatitis \\
\hline
\end{tabular}

Thirteen healthy Japanese volunteers including 10 females and 3 males were enrolled in the study. We first examined how temperament scale scores (StateTrait Anxiety Inventory-form JYZ: STAI-form JYZ, Global Scale for Depression: GSD) affect pain ratings. We show below the total score with both scales.Moreover, questions regarding the presence or absence of pain as well as stress-associated with pain were included.

shows the characteristics of the subjects.

This study was approved by the Ethics Committees of Graduate School of Health Sciences of Kobe University, the Nursing Department of Aichi Medical University and the National Institute of Communication Technology.

\subsection{Methodology}

\subsubsection{Chronic Pain Stimuli by Cold Pressor Task}

To experimentally simulate chronic pain, we used a cold pressor task (CPT) [57] scheme in which subjects are asked to immerse their hands in cold water. The temperature of the water was maintained at $10^{\circ} \mathrm{C}-11^{\circ} \mathrm{C}$ using an electrical cooling device, and this low temperature activates afferent C-fibers via TRPA1 receptors [58]. The duration of immersion was approximately a few minutes to eliminate the influence of acute pain sensation signals transmitted along the spinothalamic tract.

The C-fibers play a primary role in the neural systems for chronic pain perception, therefore, it is assumed that the CPT scheme can temporarily generate chronic or pseudo-chronic pain.

\subsubsection{Essential Oil}

We used Yuzu (Citrus junos) oil, Yuzu in Japanese, for experiments preformed in the present study. This oil has been welcomed by many Japanese who associate it with pleasant feelings. It has also been traditionally used in Japan as a popular health-maintenance remedy. Such benefits of the oil will be advantageous for making aroma touch therapy more popular in hospices or nursing homes. Taking into account the results of our advanced clinical trial as mentioned above, Yuzu oils with the same components as those used in the trial were employed in the present study. Yuzu peels (products of Kochi Prefecture, Japan) were squeezed by steam distillation under a decompression condition [59], and the wastes obtained were used for preparing Yuzu essential oil with sesame oil (Sesamum indicum; Musee Inc., Osaka, Japan). The prepared Yuzu oil consisted of linalool (70.89\%), alpha-terpineol (13.13\%), thymol (6.38\%), limonene (4.94\%), and 4-terpineol (4.66\%). The carry-over effects of the Yuzu oil was examined in advance, and throughout the examination, we did not observe any serious side effects for any subjects.

\subsubsection{Touch and Aroma Touch Therapy}

Our touch therapy was based on the superficial effleurage scheme [60,61] applied from fingers to an elbow with a stroke velocity in the range from 3 to $5 \mathrm{~cm} / \mathrm{s}$. The control touch therapy used in the study was performed according to the above procedure using a non-essential oil such as sesame oil. This scheme was followed in aroma touch therapy; however, the Yuzu essential oil was used instead of the sesame oil.

\subsubsection{Temperament Evaluation}

Subjective temperaments were evaluated using the State-Trait Anxiety Inventory (STAI)-form JYZ, modified from STAI form-Y proposed by Spielberger [62], and Global Scale for Depression (GSD) [63]. According to the STAI-JYZ criteria, scores in the range of 20 - 80 are related to specific psychiatric dysfunctions such as 
state anxiety or trait anxiety. A score of $\geq 55$ is considered high-level anxiety, and a score of $<45$ is considered low-level anxiety [64]. On the basis of the GSD criteria, depression can be objectively diagnosed according to the scores, i.e., as mild (30 - 36), moderate (37 $43)$, and serious $(\geq 44)$ [63].

\subsubsection{Objective Evaluation of Emotional Responses}

Transient emotional responses to pain were objectively evaluated using subjective reports to reveal the relationship between emotion and pain ratings. The objective evaluation was based on psychological scales including valence varying from pleasant to unpleasant, arousal varying from awaking to sleepiness, stability varying from sedation to agitation [65], vitality varying from positive to negative (fatigue), and preference to odors. A psychiatric scale related to depression or anxiety was also used to evaluate the specific emotional states of subjects under chronic pain. All scales were objectively evaluated using the Visual Analog Scale (VAS) that ranged from 0 to 100 $\mathrm{mm}$, and the VAS scores generated are sufficiently reliable for statistical analyses [66].

\subsubsection{Pain Ratings and Classification}

Pain ratings were objectively evaluated using the $\mathrm{Nu}-$ merical Rating Scale (NRS), which has been widely used in clinical practice [67]. The NRS scores ranged from 0 to 10 , and these scores are assigned according to the declaration made by the subjects on the basis of their sensitivity to pain. These NRS scores are sufficiently reliable for statistical analyses.

\subsubsection{EEG Recordings and Data Processing}

EEG recordings were performed using $21 \mathrm{Ag} / \mathrm{AgCl}$ electrodes placed according to the International 10 - 20 system (sampling frequency: $512 \mathrm{~Hz}$, resolution: $24 \mathrm{bit}$ ). EEG signals were analyzed using a fast Fourier transform (FFT) technique to generate time-series data of occipital (O1, O2) alpha-2 band $(10-13 \mathrm{~Hz})$ powers extracted from the FFT data [rate: 32-SPS (samples/s), epoch: $1 \mathrm{~s}$. The DBN activity index was defined as slow fluctuation components of the time-series data calculated by a moving-average technique.

\subsubsection{Hemoencephalogram Recordings}

As the frontal lobe is activated by pain or other unpleasant stimuli [68], we can support objective pain ratings according to a subjective declaration.

Hence, regional central blood flows at the center of a patient's forehead were measured using a near-infrared hemoencephalogram (NIR-HEG) sensor. This measurement scheme provides a simple and convenient method for evaluating the dynamic responses of regional brain activities, which can be calculated from relative oxyge- nated hemoglobin and deoxyhemoglobin concentrations. As the frontal lobe is activated by pain or other unpleasant stimuli [68], we can support objective pain ratings according to a subjective declaration.

\subsubsection{Experimental Protocol}

In a familiarization session, subjects who provided written informed consent were interviewed to know their medical history and tested not to have any allergy to essential oils used in the study. Experiments were performed with subjects who had cleared this allergy test. The experimental protocol is shown in Figure 1. For the experiment, subjects were seated upright in a comfortable chair with their eyes closed. EEG and NIR-HEG measurements were performed continuously throughout the protocol. The protocol consisted of three major sessions: a rest session for measuring baseline neural activities, a therapy session, and a pain evaluation session. The duration of each of these sessions was $5 \mathrm{~min}$. In the therapy session, touch or aroma touch therapy were applied to one hand of the subjects. In the pain evaluation session, painful stimuli through CPT were applied to the other hand of the subjects while the therapies were continued.

Pain evaluation was performed under the three conditions: a baseline condition without any therapies, a control condition using sesame oil as a non-essential oil, and an aroma touch condition using touch with the Yuzu essential oil. The subjects were asked to declare their pain at every $30 \mathrm{~s}$, and scores were accordingly assigned in the range of 0 - 10. A cross-task design was adopted to eliminate cross correlation between the control and aroma touch conditions.

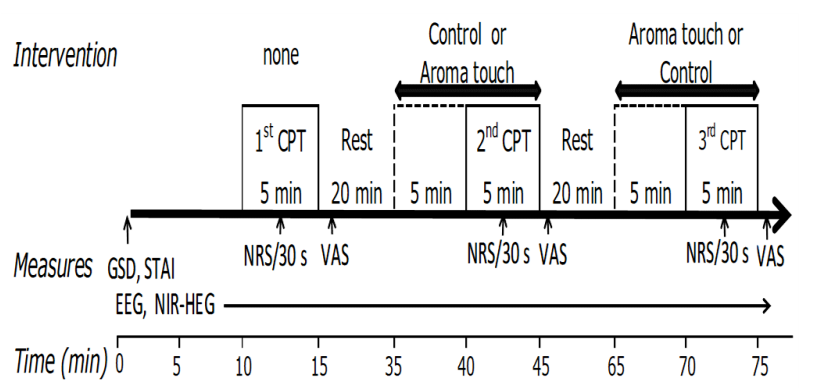

Figure 1. The study protocol. A cross-task design was adopted to eliminate the influences of the carry-over effects of essential oil. Subjects were interviewed to clarify their temperament using global scale for depression (GSD) and state-trait anxiety inventory form JYZ (STAI). Then, they were required to undergo three cold pressor task (CPT) exposures under different conditions: baseline (the baseline condition with no therapy), control [touch with placebo (sesame oil)], and aroma touch. Pain ratings were evaluated using Numerical Rating Scale (NRS) at every 30 s during CPT. Psychological evaluations for emotional responses using visual analogy scale (VAS) were performed in the rest durations. In the entire experimental period, spontaneous electroencephalogram (EEG) and near-infrared hemoencephalogram (NIR-HEG) were recorded. 


\section{Statistical Analysis}

To discuss statistical significance, we adopted paired t-test. The significance level was set at 0.05 . Calculations were performed using SPSS 20.0. To discuss statistical dependence, we used Spearman's rank correlation coefficient and Pearson's correlation coefficients. Significance was based on the critical value table where critical correlation coefficients to satisfy the significance level of 0.05 are given for each sample size.

\section{Results}

\subsection{Subjective Ratings of Chronic Pain}

Grand-averaged subjective dynamic responses to pain are shown in Figure 2(a) for the three procedural conditions (baseline, control, and aroma touch). In all of the responses, the pain ratings similarly exhibited an increase to a peak within a few minutes, and then a gradual decrease. However, pain relief dominance was maintained during the entire period under the painful stimuli such that the aroma touch condition was the first, the control condition was the second, and the baseline condition was the third.

Such dynamic responses were characterized by their maximum and final pain ratings, which were statistically analyzed (Figure 2(b)). Results show that the pain-relief effects of the aroma touch condition were always superior final pain ratings $(P<0.01)$. The control condition also exhibited similar to those of the baseline condition in both maximum and superiority in pain relief to the baseline condition $(P<0.05)$ in both maximum and final pain ratings. However, it did not exhibit any significant difference in the pain-relief effects compared with the aroma touch condition.

Because grand averages may cancel the subject-dependent specific aspects of the dynamic responses to pain, we categorized the subjects into three groups according to the final pain ratings: a strong pain relief group exhibiting final pain ratings of $0-1$, a moderate pain relief group with final pain ratings of $2-4$, and a mild a pain relief group with final pain ratings of $5-6$. The data corresponding to the final pain ratings $>7$ were not considered for this categorization. Such classification schemes according to pain ratings are considered useful for clinical practices including symptom assessment [69].

For each of the procedural conditions, dynamic responses to pain were exhibited for the three groups, as shown in Figure 2(c). Independently of the procedural conditions, the moderate and mild pain relief groups represented a response pattern similar to that for the grand averages. In contrast, the strong pain relief group exhibited extreme pain-relief effects according to the pain relief dominance as described above.
In the strong pain relief group, we found the analgesic effects of aroma touch in some subjects. These effects not only reduced maximal pain ratings but also provided complete pain-free states (NRS $=0$ ) at the end of CPT (Figure 3).

\subsection{Correlation of Pain Ratings with Temperament and Emotional Responses to Pain}

We first examined how temperament scale scores affect pain ratings. The maximum and final pain ratings versus GSD total scores are shown in Figure 4(a) for the three procedural conditions. For the aroma touch condition, a negative correlation was observed between the GSD total scores and maximum pain ratings ( $r s=-0.65, P<0.05$ ) as well as between the GSD total scores and final ratings ( $r s=-0.58, P<0.05$ ) (Figure 4(a)).

We also examined how anxiety scale scores affect pain ratings. While no correlation was observed between the trait anxiety and pain ratings, a negative correlation was observed between the state anxiety and maximum pain ratings ( $r s=-0.72, P<0.01)$ only for the control condition and a negative correlation was observed between the state anxiety and final pain ratings $(r s=-0.55, P=0.52)$ (Figure 4(b)).

Consequently, these temperamental factors exhibited a tendency to provide high pain relief effects for those who had lower averaged DBN indices, while statistical significance was completely obtained for all the factors.

We furthermore investigated emotional responses to pain. Figure 5 shows a series of emotional scale scores for the different pain relief groups. The scores were compared among the three procedural conditions. In the strong pain relief group, aroma touch seemed to induce emotional states to the condition specified by "pleasant" for the valence scale, "awaking" for the arousal scale, and "sedation" for the stability scale. In contrast, in the mild pain relief group, aroma touch seemed to induce "sedation" and "sleepiness." These emotional responses to pain did not reach any statistical significance.

\subsection{Occipital EEG Alpha-2 Power Responses to Chronic Pain Stimuli}

In a typical dynamic response to chronic pain imposed by the painful stimuli administered in CPT, the occipital EEG alpha-2 power as a DBN activity index exhibited a transient sharp increase to a peak within a few minutes after CPT began, and then a prompt decrease, as shown in Figure 6.

To objectively evaluate the correlation between painrelief effects and DBN activity, we adopted an average DBN activity index, which is defined as an overall DBN 


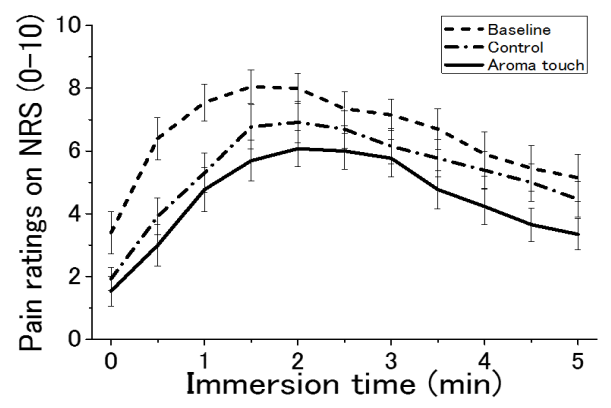

(a)
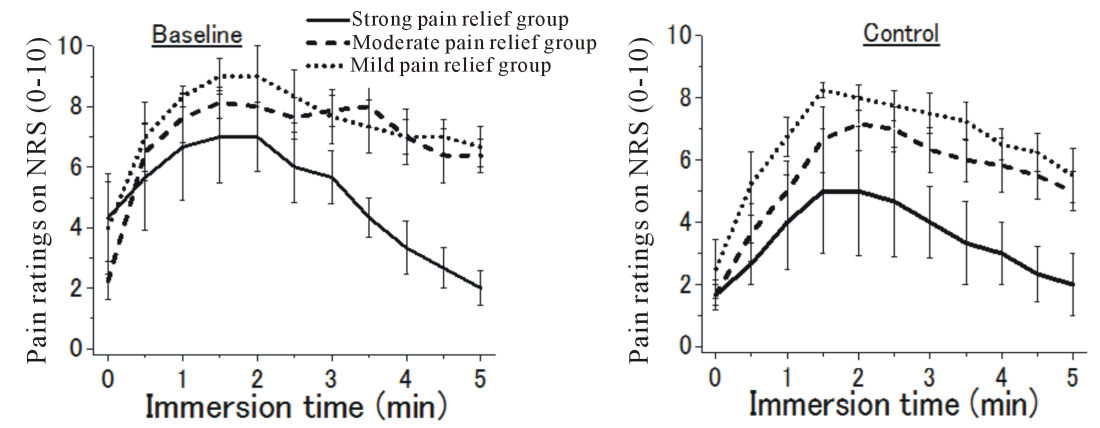

(c)

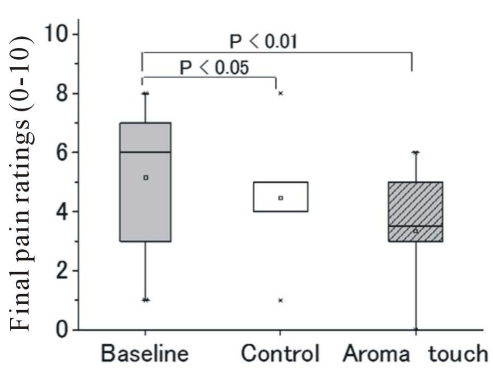

(b)

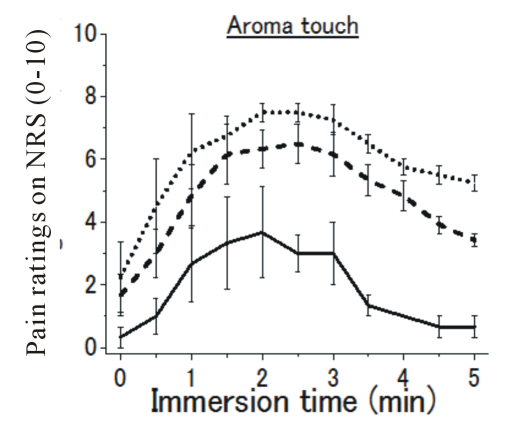

Figure 2. General aspects of changes in pain rating during cold pressor task (CPT) under the three procedural conditions of the baseline, control, and aroma touch. Pain ratings were numerically evaluated using the numerical rating scale (NRS), which ranged from 0 to 10 , and these scores are assigned according to the declaration made by the subjects. (a) Grand-averaged temporal changes in pain ratings(mean \pm standard error). (b) Test of significance in pain-relief effects for the three procedural conditions evaluated using maximum and final pain rating scores in the temporal changes in pain rating (paired t-test). (c) Individual evaluations of temporal changes in pain rating during CPT duration for the three groups: a strong pain relief group (final pain ratings in a range of 0 - 2), a moderate pain relief group ( 3 - 4), and mild pain relief group (5 - 6). Data of a group with pain ratings of $>7$ were not used for this evaluation because the group did not show any pain-relief effects compared with their initial pain ratings when CPT began (mean \pm standard error).

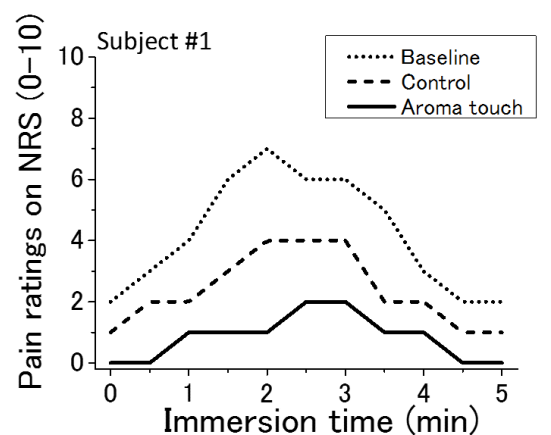

Figure 3. Temporal changes in pain rating exhibited by an extraordinary case that developed complete pain-free states (Analgesia) in the final stage of the cold pressor task. Pain ratings under the three conditions in a subject from the strong pain relief group.

activity derived from the occipital EEG alpha-2 rhythm powers processed by the moving average technique during the 5-min CPT. Figure 7 shows the maximum and final pain ratings as a function of the average DBN activity index. In each of these two cases, we found extraordinary correlations exhibiting a V-shaped curve in the maximum/final pain ratings versus the average DBN activity index. Such correlations were attributed to the fact that the effects of chronic pain relief were positively correlated with DBN activity in the regions with lower DBN activities, whereas the effects were negatively correlated with DBN activity in the regions with higher DBN activities. These correlations provided higher absolute correlation coefficients $(r>0.8)$ with significance $(P<0.001)$ particularly for the final pain ratings.

\subsection{Neurophysiological Evaluation of a Complete Pain-Free State Induced by Aroma Touch Therapy}

We found that a complete pain-free state can be obtained by aroma touch therapy, as shown in Figure 3. We here measured neurophysiological responses under such an analgesic state. Figure 8 shows the dynamic responses of DBN activity evaluated by occipital EEG alpha-2 rhythm powers and the corresponding regional blood flows at the center of a subject's forehead evaluated by NIR-HEG for the three procedural conditions. For both the baseline and control conditions, DBN activity remained lower, while 

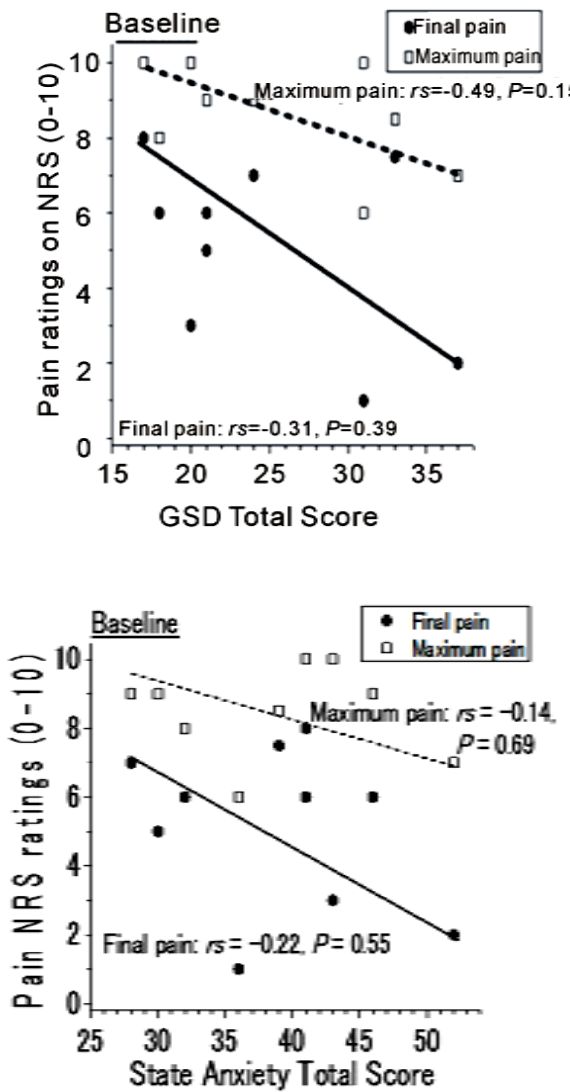

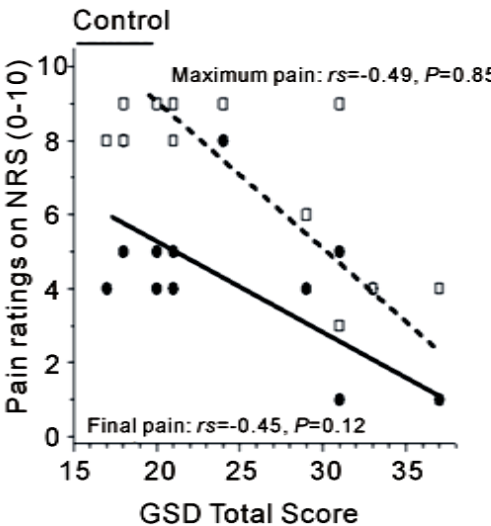

(a)

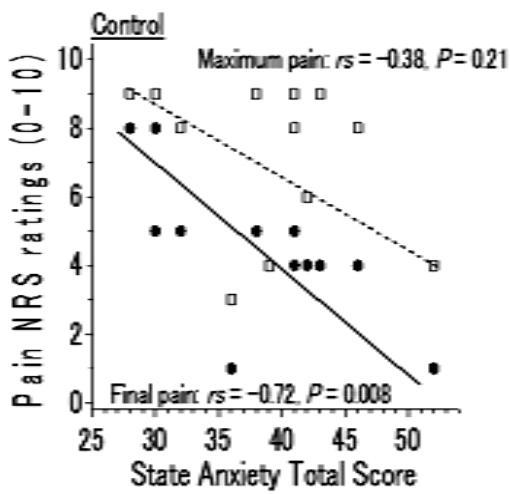

(b)
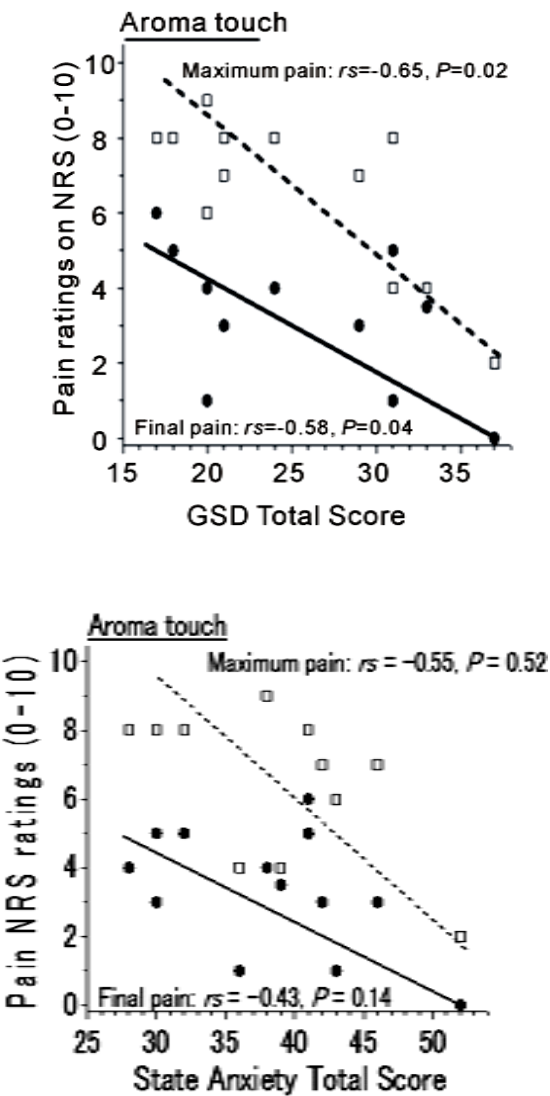

Figure 4. Temperament scale dependences on maximum and final pain ratings, which characterize pain ratings during the Cold Pressor Task. The maximum and final pain ratings under the three conditions (baseline, control, and aroma touch) examined the correlation between the Global Scale for Depression (GSD) total score and the state anxiety total score (Spearman's rank correlation coefficient. (a) Correlation between GSD and the pain, (b) Correlation between the state anxiety of State-Trait Anxiety Inventory (STAI)-form JYZ and the pain.
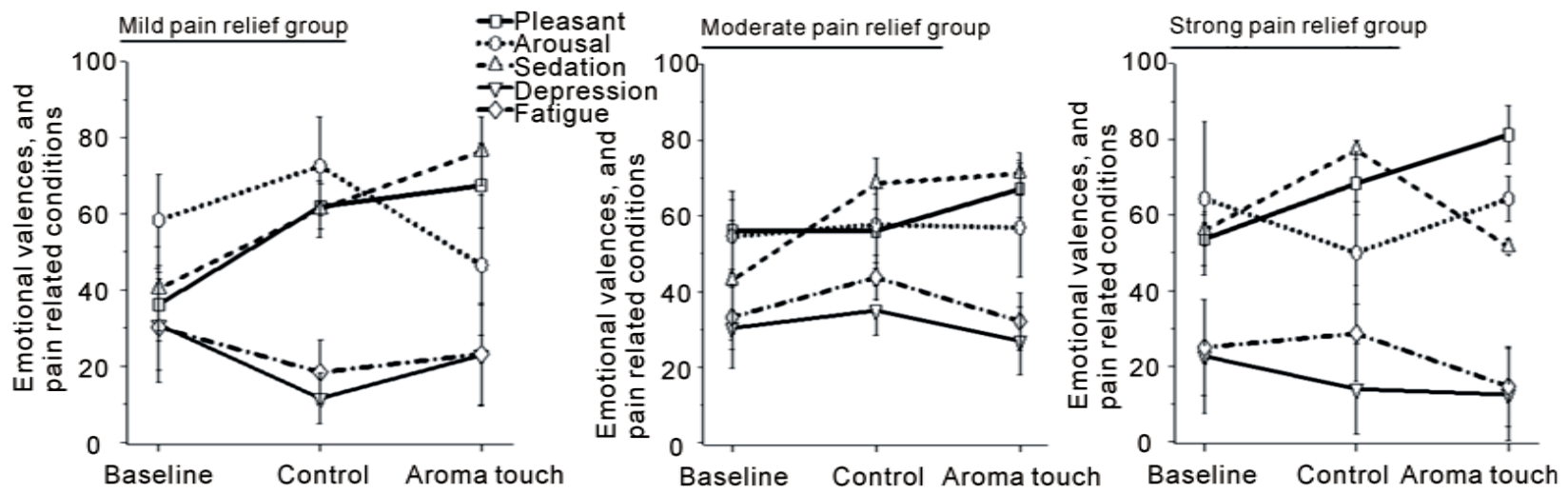

Figure 5. Evaluation of emotional and temperament states during the cold pressor task for the three subject groups. The graphs display a strong pain relief group (final pain ratings of 0 - 2), a moderate pain relief group ( 3 - 4), and a mild pain relief group (5 - 6). The emotional states were evaluated using three emotional scales, including valence (from pleasant to unpleasant), arousal (from awaking to sleepiness), and stability (from sedation to agitation), and two temperament scales related to depression and fatigue. No significance was observed was between the emotional/temperament scales and procedural conditions.

NIR-HEG responses exhibited an increase and remained higher after CPT began. Such NIR-HEG responses indicated that the subjects surely felt pain. In contrast, for the aroma touch condition, DBN activity exhibited an extreme increase and the corresponding NIR-HEG responses exhibited a significant decrease. The lower NIR- 


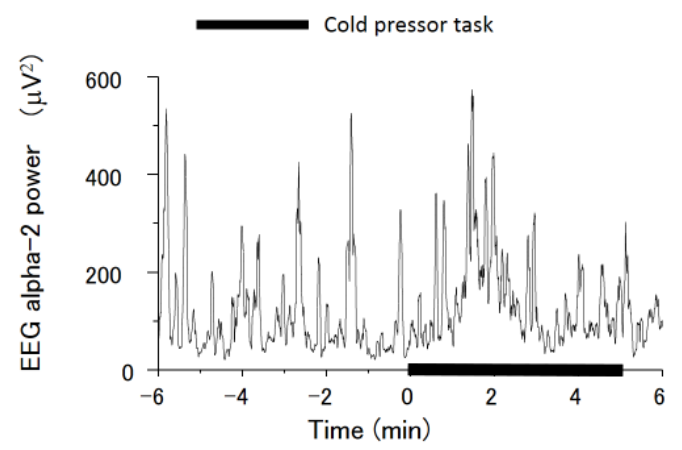

Figure 6. A Typical dynamic response of the occipital EEG alpha-2 (10 - $13 \mathrm{hz})$ rhythm powers under the 5-min cpt painful stimuli.
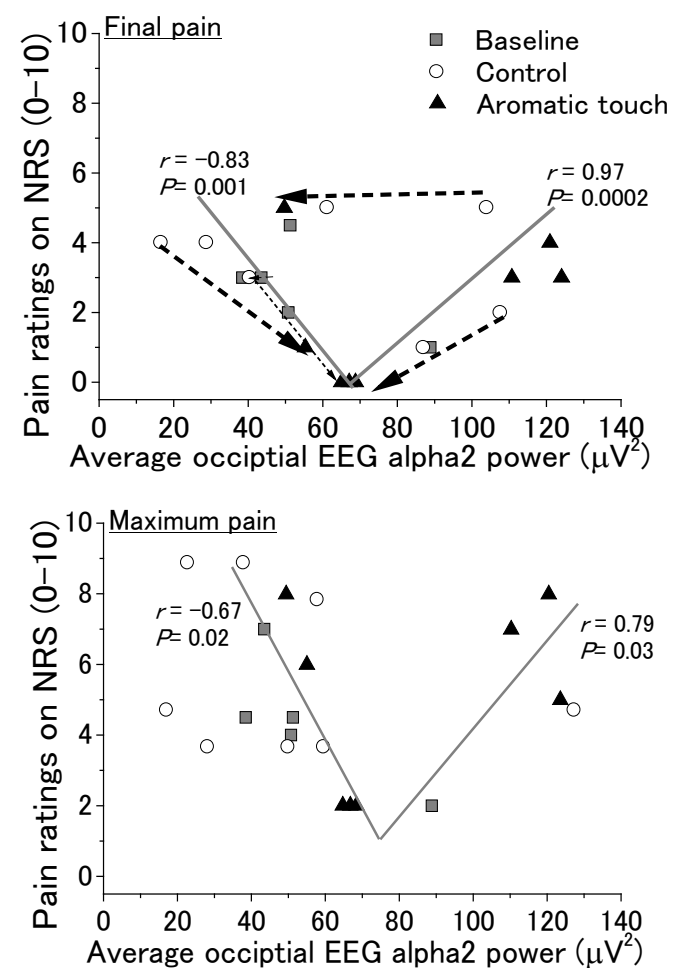

Figure 7. DBN activity evaluated by occipital EEG alpha-2 rhythm powers versus pain relief performances evaluated by maximum and final pain ratings during the cold pressor task. This figure shows the maximum and final pain ratings as a function of the average DBN activity index. Data from all subjects were superposed in the graphs, in which strong negative and positive correlations were found (Pearson's correlation coefficients). The arrow shows the movement toward the direction that was believed to be the personal equivalent point.

HEG confirmed that the subjects did not feel any pains.

\section{Discussion}

We investigated the pain-relief effects of aroma touch therapy with Citrus junos oil for cold pressure-induced chronic pain. We found a strong correlation between the pain-relief effects and DBN activity that was estimated by occipital EEG alpha-2 rhythm powers independently of emotional changes. We also found that aroma touch therapy optimally modulates DBN activity to obtain maximal analgesic effects.

\subsection{How DBN Activity Is Changed by the Pain-Relief Effects of Aroma Touch Therapy?}

The results of the positive correlation between the pain ratings and the DBN activity index agreed with the prediction by the previous studies on activation of the thalamus for chronic pain relief. However, the negative correlation was not expected based on the hypothesis. Many factors may contribute to providing such characteristics of the correlation. A promising factor is the dual pathway for transmitting signals of chronic pain. Nociceptive pain stimuli may activate an acute pain pathway related to the spinothalamic tract well as a dull pain pathway related to thalamic intralaminar nuclei. The former deteriorates pain-relief effects, while the latter alleviates chronic pain sensations, as we expect. The total pain ratings evaluated by the sum of the two pain sensations represents such V-shaped curves.

We also found that aroma touch therapy did not always increase DBN activity. This means that DBN activity was increased in the region with lower DBN activity, while it was decreased in the region with higher DBN activity. However, pain ratings were always decreased along the V-shaped curve by aroma touch therapy. Although such DBN behaviors may be explained on the basis of the balance of monoaminergic systems, the true mechanism must be clarified in future studies.

\subsection{Which Is Dominant, DBN Activity or Emotional Effects in Chronic Pain Relief?}

While correlations between emotional responses and pain ratings were consistent for the three procedural conditions (baseline, control, and aroma touch), strong correlations between DBN activity and pain ratings, exhibiting a V-shaped curve, were observed. These findings indicate that DBN activity is dominant for chronic pain relief independently of emotional responses. Emotional responses changing to "pleasant" and "awaking" are non-essential for pain relief and are merely associated with increasing DBN activity.

\section{Limitations}

The study sample was small and lacked homogeneity of subjects. Notwithstanding these limitations, the neural mechanism of chronic pain relief based on DBN activity, as indicated by the findings, will be essential for predicting subject-dependent pain relief performances. Further 

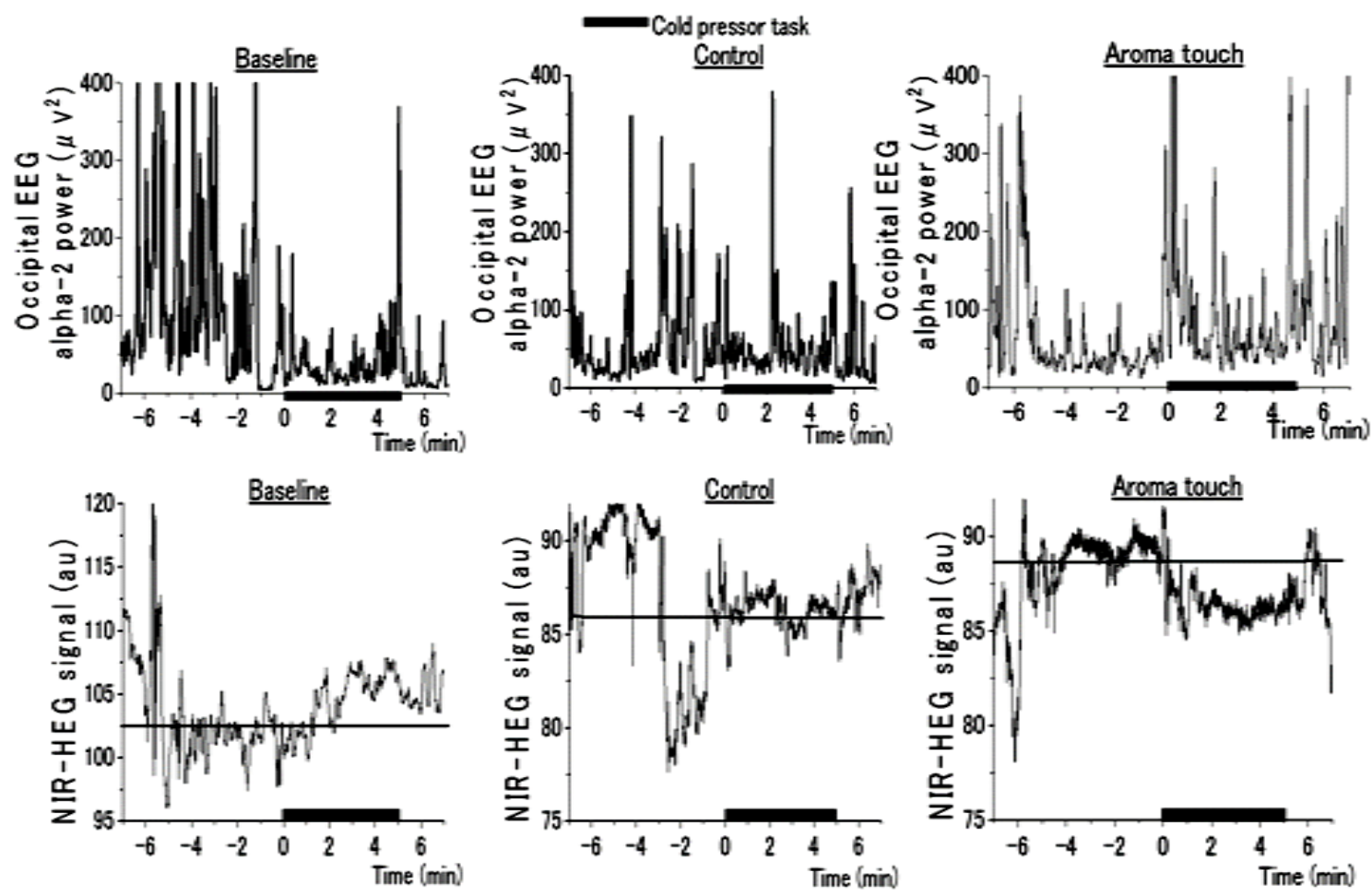

Figure 8. Dynamic responses of DBN activity evaluated by Occipital EEG Alpha-2 Rhythm Powers and corresponding regional blood flows evaluated by Near-infrared Hemoencephalogram (NIR-HEG) signals during the Cold Pressor Task (CPT). The $\mathrm{X}$-axis represents time in min, the closed bar on the $\mathrm{X}$-axis indicates the duration of $\mathrm{CPT}$, and 0 min indicates the starting point of CPT.

studies of such mechanisms should aim to determine methods to relieve chronic pain in cancer patients.

\section{Conclusions}

We investigated the analgesic effects of aroma touch therapy. We found that the pain-relief effects are strongly correlated with DBN activity, and are evaluated on the basis of the slow fluctuation components of occipital EEG alpha-2 $(10-13 \mathrm{~Hz})$ powers independent of emotional changes. We found that the correlation exhibits a V-shaped curve; that the pain ratings decrease with increasing DBN activity in the region with lower DBN activity, while they increase with decrease in DBN activity in the region with higher DBN activity. We also found that the V-shaped curve provides an optimum condition that can induce complete pain-free states.

Notwithstanding that neural mechanisms must be elicited for explaining such a strong correlation between DBN activity and the pain-relief effects, objective evaluation scheme using the DBN activity will be a powerful tool for investigating a wide variety of clinical effects of aroma or aroma touch therapies.

\section{Conflict of Interests}

The authors declare that they have no conflict of inter- ests.

\section{Acknowledgements}

This work was partially supported by Grants-in-Aid for Scientific Research (23593274, 25420236) and New Energy and Industrial Technology Development Organization promoted by Japan Society for the Promotion of Science.

\section{REFERENCES}

[1] S. R. Adler and J. R. Fosket, "Disclosing Complementary and Alternative Medicine Use in the Medical Encounter: A Qualitative Study in Women with Breast Cancer," The Journal of Family Practice, Vol. 48, No. 6, 1999, pp. 453-458.

[2] J. Buckle, "Clinical Aromatherapy: Essential Oils in Practice, 2e,” Churchill Livingstone, Livingstone, 2003.

[3] B. R. Cassileth, G. E. Deng, J. E. Gomez, P. A. J. stone, N. Kumarand A. J. Vickers, "American College of Chest Physicians. Complementary Therapies and Integrative Oncology in Lung Cancer: ACCP Evidence Based Clinical Practice Guidelines (2nd Edition)," Chest, Vol. 132, No. 3, 2007, pp. 340-354.

[4] R. Srivastava, "What's the Alternative? The World Wide Web of Integrative Medicine,” The New England Journal of Medicine, Vol. 366, No. 9, 2012, pp. 783-785. 
http://dx.doi.org/10.1056/NEJMp1110812

[5] D. Jimbo, Y. Kimura, M. Taniguchi, M. Inoue and K. Urakami, "Effect of Aromatherapy on Patients with Alzheimer's Disease,” Psychogeriatrics, Vol. 9, No. 4, 2009, pp. 173-179. http://dx.doi.org/10.1111/j.1479-8301.2009.00299.x

[6] S. Price and L. Price, "Aromatherapy for Health Professionals, 3e,” 4th Edition, Churchill Livingstone, Livingstone, 2011, pp. 61-62.

[7] J. K. Fung, H. W. Tsang and R. C. Chung, "A Systematic Review of the Use of Aromatherapy in Treatment of Behavioral Problems in Dementia," Geriatrics \& Gerontology International, Vol. 12, No. 13, 2012, pp. 372-382. http://dx.doi.org/10.1111/j.1447-0594.2012.00849.x

[8] C. Y. Fu, W. Moyle and M. Cooke, “A Randomised Controlled Trial of the Use of Aromatherapy and Hand Massage to Reduce Disruptive Behaviour in People with Dementia,” BMC Complementary and Alternative Medicine, Vol. 13, 2013, p. 165.

http://dx.doi.org/10.1186/1472-6882-13-165

[9] B. Johannessen, "Nurses Experience of Aromatherapy Use with Dementia Patients Experiencing Disturbed Sleep Patterns. An Action Research Project," Complementary Therapies in Clinical Practice, Vol. 19, No. 4, 2013, pp. 209-213. http://dx.doi.org/10.1016/j.ctcp.2013.01.003

[10] D. Sinha and D. Efron, "Complementary and Alternative Medicine Use in Children with Attention Deficit Hyperactivity Disorder,” Journal of Paediatrics and Child Health, Vol. 41, No. 1-2, 2005, pp. 23-26. http://dx.doi.org/10.1111/j.1440-1754.2005.00530.x

[11] N. Morris, S. Birtwistle and M. Toms, "Anxiety Reduction by Aromatherapy: Anxiolytic Effects of Inhalation of Geranium and Rosemary,” International Journal of Aromatherapy, Vol. 7, 1995, pp. 33-39. http://dx.doi.org/10.1016/0962-4562(95)80038-7

[12] P. Conrad and C. Adams, "The Effects of Clinical Aromatherapy for Anxiety and Depression in the High Risk Postpartum Woman: A Pilot Study," Complementary Therapies in Clinical Practice, Vol. 18, No. 3, 2012, pp. 164-168. http://dx.doi.org/10.1016/j.ctcp.2012.05.002

[13] T. Matsumoto, H. Asakura and T. Hayashi, "Does Lavender Aromatherapy Alleviate Premenstrual Emotional Symptoms? A Randomized Crossover Trial,” MedBioPsychoSocial Medicine, Vol. 7, No. 1, 2013, p. 12. http://dx.doi.org/10.1186/1751-0759-7-12

[14] J. Buckle, "Use of Aromatherapy as a Complementary Treatment for Chronic Pain,” Alternative Therapies in Health and Medicine, Vol. 5, No. 5, 1999, pp. 42-51.

[15] M. S. Ju, S. Lee, I. Bae, M. H. Hur, K. Seong and M. S. Lee, "Effects of Aroma Massage on Home Blood Pressure, Ambulatory Blood Pressure, and Sleep Quality in Middle-Aged Women with Hypertension,” Evidence-Based Complementary and Alternative Medicine, 2013, Article ID: 403251. http://dx.doi.org/10.1155/2013/403251

[16] S. M. Peng, M. Koo andZ. R. Yu, "Effects of Music and Essential Oil Inhalation on Cardiac Autonomic Balance in Healthy Individuals," Journal of Alternative and Complementary Medicine, Vol. 15, No. 1, 2009, pp. 53-57. http://dx.doi.org/10.1089/acm.2008.0243
[17] B. Wiegand, K. Luedtke, D. Friscia, M. Nair, M. Aleles and R. McCloskey, "Efficacy of a Comprehensive Program for Reducing Stress in Women: A Prospective, Randomized Trial," Current Medical Research and Opinion, Vol. 26, No. 4, 2010, pp. 991-1002. http://dx.doi.org/10.1185/03007991003688193

[18] S. Kim, H. J. Kim, J. S. Yeo, S. J. Hong, J. M. Lee and Y. Jeon, "The Effect of Lavender Oil on Stress, Bispectralindex Values, and Needle Insertion Pain in Volunteers," Journal of Alternative and Complementary Medicine, Vol. 17, No. 9, 2011, pp. 823-826. http://dx.doi.org/10.1089/acm.2010.0644

[19] S. M. Downer, M. M. Cody, P. McCluskey, P. D. Wilson, S. J. Arnott, T. A. Lister and M. L. Slevin, "Pursuit and Practice of Complementary Therapies by Cancer Patients Receiving Conventional Treatment,” BMJ, Vol. 309, No. 6947, 1994, pp. 86-89. http://dx.doi.org/10.1136/bmj.309.6947.86

[20] K. Soden, K. Vincent, S. Craske, C. Lucas and S. Aahley, "A Randomized Controlled Trial of Aromatherapy Massage in a Hospice Setting,” Palliative Medicine, Vol. 18, No. 2, 2004, pp. 87-92. http://dx.doi.org/10.1191/0269216304pm874oa

[21] D. Fellowes, K. Barnes and S. Wilkinson, “Aromatherapy and Massage for Symptom Relief in Patients with Cancer," Cochrane Database of Systematic Reviews, Vol. 2, 2004, Article ID: CD002287.

[22] D. Boyd, K. Merkh, D. N. Rutledge and V. Randall, "Nurses' Perceptions and Experiences with End-of-Life Communication and Care," Oncology Nursing Forum, Vol. 30, No. 3, 2011, pp. 229-239. http://dx.doi.org/10.1188/11.ONF.E229-E239

[23] K. Boehm, A. Büssing and T. Ostermann, “Aromatherapy as an Adjuvant Treatment in Cancer Care: A Descriptive Systematic Review," African Journal of Traditional, Complementary and Alternative Medicine, Vol. 9, No. 4, 2012, pp. 503-518. http://dx.doi.org/10.4314/ajtcam.v9i4.7

[24] J. Corner, N. Cawley and S. Hildebrand, “An Evaluation of the Use of Massage and Essential Oils in the Wellbeing of Cancer Patients," International Journal of Palliative Nursing, Vol. 1, 1995, pp. 67-73.

[25] S. Y. Chang, "Effects of Aroma Hand Massage on Pain, State Anxiety and Depression in Hospice Patients with Terminal Cancer,” Taehan Kanho Hakhoe Chi, Vol. 38, No. 4, 2008, pp. 493-502.

http://dx.doi.org/10.4040/jkan.2008.38.4.493

[26] K. Nakano, K. Sato, H. Katayama and M. Miyashita, "Living with Pleasure in Daily Life at the End of Life: Recommended Care Strategy for Cancer Patients from the Perspective of Physicians and Nurses," Palliat Support Care, 2012, pp. 1-9.

[27] L. Berger, M. Tavares and B. Berger, “A Canadian Experience of Integrating Complementary Therapy in a Hospital Palliative Care Unit,” Journal of Palliative Medicine, Vol. 16, No. 10, 2013, pp. 1294-1298. http://dx.doi.org/10.1089/jpm.2013.0295

[28] S. Beider and C. A. Moyer, "Randomized Controlled Trials of Pediatric Massage: A Review,” Evidence-Based Complementary and Alternative Medicine, Vol. 4, No. 1, 
2007, pp. 23-34. http://dx.doi.org/10.1093/bja/77.6.727

[29] L Corbin, "Safety and Efficiency of Massage Therapy for Patients with Cancer," Cancer Control, Vol. 12, No. 3, 2005, pp. 158-164.

[30] S. K. Hamid, N. McCann, L. McArdle and A. J. Asbury, "Comparison of Patient-Controlled Sedation with Either Methohexitone or Propofol," British Journal of Anaesthesia, Vol. 77, No. 6, 1996, pp. 727-730. http://dx.doi.org/10.1093/bja/77.6.727

[31] S. Wilkinson, J. Aldridge, I. Salmon, E. Cain and B. Wilson, "An Evaluation of Aromatherapy Massage in Palliative Care,” Palliative Medicine, Vol. 13, No. 5, 1999, pp. 409-417. http://dx.doi.org/10.1191/026921699678148345

[32] J. S. Kutner, M. C. Smith, L. Corbin, L. Hemphill, K. Benton, B. K. Mellis, B. Beaty, S. Felton, T. E. Yamashita, L. L. Bryant and D. L. Fairclough, "Massage Therapy versus Simple Touch to Improve Pain and Mood in Patients with Advanced Cancer: A Randomized Trial," Annals of Internal Medicine, Vol. 149, No. 6, 2008, pp. 369379.

http://dx.doi.org/10.7326/0003-4819-149-6-200809160-0 $\underline{0003}$

[33] T. Bohgaki, "Effects of Aroma Touch to a Patient with Multiple-Organ Metastasis after Rectal Cancer Surgery," The 33rd Academic Conference of JAPAN Academy of Nursing Science, Osaka, 2013, p. 398. (Japanese)

[34] R. Melzack, "Phantom Limbs and the Concept of a Neuromatrix,” Trends in Neurosciences, Vol. 13, No. 3, 1990, pp. 88-92. http://dx.doi.org/10.1016/0166-2236(90)90179-E

[35] D. R. Kenshalo Jr., G. J. Giesler Jr., R. B. Leonard and W. D. Willis, "Responses of Neurons in Primate Ventral Posterior Lateral Nucleus to Noxious Stimuli,” Journal of Neurophysiology, Vol. 43, No. 6, 1980, pp. 1594-1614.

[36] T. Ushida, M. Fukumoto, C. Binti, T. Ikemoto, S. Taniguchi, M. Ikeuchi, M. Nishihara and T. Tani, "Alterations of Contralateral Thalamic Perfusion in Neuropathic Pain," The Open Neuroimaging Journal, Vol. 4, 2010, pp. 182186.

[37] V. Di Piero, A. K. Jones, F. Iannotti, M. Powell, D. Perani, G. L. Lenzi and R. S. Frackowiak, "Chronic Pain: A PET Study of the Central Effects of Percutaneous High Cervical Cordotomy,” Pain, Vol. 46, No. 1, 1991, pp. 912. http://dx.doi.org/10.1016/0304-3959(91)90026-T

[38] M. J. Iadarola, M. B. Max, K. F. Berman, M. G. ByasSmith, R. C. Coghill, R. H. Gracely and G. J. Bennett, "Unilateral Decrease in Thalamic Activity Observed with Positron Emission Tomography in Patients with Chronic Neuropathic Pain,” Pain, Vol. 63, No. 1, 1995, pp. 55-64. http://dx.doi.org/10.1016/0304-3959(95)00015-K

[39] R. C. Kupers, J. M. Gybels and A. Gjedde, "Positron Emission Tomography Study of a Chronic Pain Patient Successfully Treated with Somatosensory Thalamic Stimulation,” Pain, Vol. 87, No. 3, 2000, pp. 295-302. http://dx.doi.org/10.1016/S0304-3959(00)00295-5

[40] U. Ribary, A. A. Ioannides, K. D. Singh, R. Hasson, J. P. Bolton, F. Lado, A. Mogilner and R. Llinas, "Magnetic Field Tomography of Coherent Thalamocortical $40-\mathrm{Hz}$ Oscillations in Humans," Proceedings of the National
Academy of Sciences of United States of America, Vol. 88, No. 24, 1991, pp. 11037-11041.

[41] R. R. Llinás, U. Ribary, D. Jeanmonod, E. Kronberg and P. P. Mitra, "Thalamocortical Dysrhythmia: A Neurological and Neuropsychiatric Syndrome Characterized by Magnetoencephalography," Proceedings of the National Academy of Sciences of United States of America, Vol. 96, No. 26, 1999, pp. 15222-15227. http://dx.doi.org/10.1073/pnas.96.26.15222

[42] J. Stern, D. Jeanmonod and J. Sarnthein, "Persistent EEG Overactivation in the Cortical Pain Matrix of Neurogenic Pain Patients,” Neuroimage, Vol. 31, No. 2, 2006, pp. 721-731.

[43] G. D. Iannetti and A. Mouraux, "From the Neuromatrix to the Pain Matrix (and Back),” Experimental Brain Research, Vol. 205, No. 1, 2010, pp. 1-12. http://dx.doi.org/10.1007/s00221-010-2340-1

[44] M. A. Farmer, M. N. Baliki and A. V. Apkarian, "A Dynamic Network Perspective of Chronic Pain,” Neuroscience Letters, Vol. 520, No. 2, 2012, pp. 197-203. http://dx.doi.org/10.1016/j.neulet.2012.05.001

[45] L. Garcia-Larrea and R. Peyron, "Pain Matrices and Neuropathic Pain Matrices: A Review,” Pain, Vol. 154, Suppl. 1, 2013, pp. S29-S43. http://dx.doi.org/10.1016/j.pain.2013.09.001

[46] S. Kamping, I. C. Bomba, P. Kanske, E. Diesch and H. Flor, "Deficient Modulation of Pain by a Positive Emotional Context in Fibromyalgia Patients,” Pain, Vol. 154, No. 9, 2013, pp. 1846-1855. http://dx.doi.org/10.1016/j.pain.2013.06.003

[47] J. A. Hashmi, A. T. Baria, M. N. Baliki, L. Huang, T. J. Schnitzer and A. V. Apkarian, "Brain Networks Predicting Placebo Analgesia in a Clinical Trial for Chronic Back Pain,” Pain, Vol. 153, No. 12, 2012, pp. 2393-2402. http://dx.doi.org/10.1016/j.pain.2012.08.008

[48] Y. Masaoka, M. Takayama, H. Yajima, A. Kawase, N. Takakura and I. Homma, “Analgesia Is Enhanced by Providing Information Regarding Good Outcomes Associated with an Odor: Placebo Effects in Aromatherapy?” Evidence-Based Complementary and Alternative Medicine, Vol. 2013, Article ID: 921802.

[49] L. Colloca, R. Klinger, H. Flor and U. Bingel, "Placebo Analgesia: Psychological and Neurobiological Mechanisms,” Pain, Vol. 154, No. 4, 2013, pp. 511-514. http://dx.doi.org/10.1016/j.pain.2013.02.002

[50] N. Sadato, S. Nakamura, T. Oohashi, E. Nishina, Y. Fuwamoto, A. Waki and Y. Yonekura, "Neural Networks for Generation and Suppression of Alpha Rhythm: A PET Study," NeuroReport, Vol. 9, No. 5, 1998, pp. 893-897. http://dx.doi.org/10.1097/00001756-199803300-00024

[51] R. I. Goldman, J. M. Stern, J. Engel Jr. and M. S. Cohen, "Simultaneous EEG and fMRI of the Alpha Rhythm," NeuroReport, Vol. 13, No. 18, 2002, pp. 2487-2492. http://dx.doi.org/10.1097/00001756-200212200-00022

[52] M. Schreckenberger, C. Lange-Asschenfeldt, M. Lochmann, K. Mann, T. Siessmeier, H. G. Buchholz, P. Bartenstein and G. Gründer, “The Thalamus as the Generator and Modulator of EEG Alpha Rhythm: A Combined PET/EEG Study with Lorazepam Challenge in Humans,” 
Neuroimage, Vol. 22, No. 2, 2004, pp. 637-644. http://dx.doi.org/10.1016/j.neuroimage.2004.01.047

[53] C. L. Larson, R. J. Davidson, H. C. Abercrombie, R. T. Ward, S. M. Schaefer, D. C. Jackson, J. E. Holden and S. B. Perlman, "Relations between PET-Derived Measures of Thalamic Glucose Metabolism and EEG Alpha Power," Psychophysiology, Vol. 35, No. 2, 1998, pp. 162-169. http://dx.doi.org/10.1111/1469-8986.3520162

[54] K. A. Lindgren, C. L. Larson, S. M. Schaefer, H. C. Abercrombie, R. T. Ward, T. R. Oakes, J. E. Holden, S. B. Perlman, R. M. Benca and R. J. Davidson, “Thalamic Metabolic Rate Predicts EEG Alpha Power in Healthy Control Subjects but not in Depressed Patients,” Biological Psychiatry, Vol. 45, No. 8, 1999, pp. 943-952. http://dx.doi.org/10.1016/S0006-3223(98)00350-3

[55] T. Oohashi, E. Nishina, M. Honda, Y. Yonekura, Y. Fuwamoto, N. Kawai, T. Maekawa, S. Nakamura, H. Fukuyama and H. Shibasaki, "Inaudible Highfrequency Sounds Affect Brain Activity: Hypersonic Effect,” Journal of Neurophysiology, Vol. 83, No. 6, 2000, pp. 3548-3558.

[56] K. Omata, T. Hanakawa, M. Morimoto and M. Honda, "Spontaneous Slow Fluctuation of EEG Alpha Rhythm Reflects Activity in Deep-Brain Structures: A Simultaneous EEG-fMRI Study,” PLoS ONE, Vol. 8, No. 6, 2013, Article ID: e66869. http://dx.doi.org/10.1371/journal.pone.0066869

[57] K. A. Birnie, M. Petter, K. E. Boerner, M. Noel and C. T. Chambers, "Contemporary Use of the Cold Pressor Task in Pediatric Pain Research: A Systematic Review of Methods,” The Journal of Pain, Vol. 13, No. 9, 2012, pp. 817-826. http://dx.doi.org/10.1016/j.jpain.2012.06.005

[58] D. Uta, H. Furue, A. E. Pickering, M. H. Rashid, H. Mizuguchi-Takase, T. Katafuchi, K. Imoto and M. Yoshimura, "TRPA1-Expressing Primary Afferents Synapse with a Morphologically Identified Subclass of Substantia-Gelatinosa Neurons in the Adult Rat Spinal Cord," European Journal of Neuroscience, Vol. 31, No. 11, 2010, pp. 1960-1973.

http://onlinelibrary.wiley.com/doi/10.1111/j.1460-9568.2 010.07255.x/full http://dx.doi.org/10.1111/j.1460-9568.2010.07255.x

[59] M. Sawamura, "Essential Oil Extraction," Patent No. 3842794. (Japan)

[60] M. Nordin, "Low-Threshold Mechanoreceptive and Nociceptive Units with Unmyelinated (C) Fibers in the Human Supraorbital Nerve," The Journal of Physiology, Vol. 426, 1990, pp. 229-240. http://jp.physoc.org/content/426/1/229.long

[61] L. S. Löken, J. Wessberg, I. Morrison, F. McGlone and H. Olausson, "Coding of Pleasant Touch by Unmyelinated Afferents in Humans," Nature Neuroscience, Vol. 12, No. 5, 2009, pp. 547-548. http://dx.doi.org/10.1038/nn.2312

[62] C. D. Spielberger, R. L. Gorsuch, P. R. Lushene, P. R. Vagg and A. G. Jacobs, "Manual for the State-Trait Anxiety Inventory (Form Y)," Consulting Psychologists Press, Inc., Palo Alto, 1983.

[63] I. Hukunishi, "Global Scale for Depression Manual," Chiba Test Center, Japan.

[64] T. Hidano, M. Hukuhara and S. Iwawaki, "State-Trait Anxiety Inventory-Form JYZ Manual,” Jitsumukyouikusuppan, 2009. (Japanese)

[65] J. A. Russell and L. F. Barrett, "Core Affect, Prototypical Emotional Episodes, and Other Things Called Successfully Treated with Somatosensory Thalamic Stimulation,” Pain, Vol. 87, No. 3, 2000, pp. 295-302.

[66] S. Anders, M. Lotze, M. Erb, W. Grodd and N. Birbaumer, "Brain Activity Underlying Emotional Valence and Arousal: A Response-Related fMRI Study," Human Brain Mapping, Vol. 23, No. 4, 2004, pp. 200-209. http://onlinelibrary.wiley.com/doi/10.1002/hbm.20048/pd f http://dx.doi.org/10.1002/hbm.20048

[67] A. Williamson and B. Hoggart, "Pain: A Review of Three Commonly Used Pain Rating Scales,” Journal of Clinical Nursing, Vol. 14, No. 7, 2005, pp. 798-804. http://dx.doi.org/10.1111/j.1365-2702.2005.01121.x

[68] C. H. Lee, T. Sugiyama, A. Kataoka, A. Kudo, F. Fujino, Y. W. Chen, Y. Mitsuyama, S. Nomura and T. Yoshioka, "Analysis for Distinctive Activation Patterns of Pain and Itchy in the Human Brain Cortex Measured Using Near Infrared Spectroscopy (NIRS),” PLoS ONE, Vol. 8, No. 10, 2013, Article ID: e75360.

[69] B. Given, C. W. Given, A. Sikorskii, S. Jeon, R. McCorkle, V. Champion and D. Decker, "Establishing Mild, Moderate, and Severe Scores for Cancer Related Symptoms: How Consistent and Clinically Meaningful Are Interference. Based Severity Cutpoints?” Journal of Pain and Symptom Management, Vol. 35, No. 2, 2008, pp. 126-135.

http://www.sciencedrect.com/scence/artcle/pii/S08853924 $\underline{0700669}$ 